Mots. Les langages du politique

$80 \mid 2006$

La politique mise au net

\title{
Les blogs et leurs usages politiques lors de la campagne présidentielle de 2004 aux États-Unis
}

Viviane Serfaty

\section{(2) OpenEdition}

Journals

Édition électronique

URL : https://journals.openedition.org/mots/501

DOI : $10.4000 /$ mots.501

ISSN : 1960-6001

Éditeur

ENS Éditions

Édition imprimée

Date de publication : 1 mars 2006

Pagination : 25-35

ISBN : 2-84788-095-X

ISSN : 0243-6450

Référence électronique

Viviane Serfaty, «Les blogs et leurs usages politiques lors de la campagne présidentielle de 2004 aux États-Unis », Mots. Les langages du politique [En ligne], 80 | 2006, mis en ligne le 01 mars 2008, consulté le 23 avril 2022. URL : http://journals.openedition.org/mots/501 ; DOI : https://doi.org/ $10.4000 /$ mots. 501 


\section{Les blogs et leurs usages politiques lors de la campagne présidentielle de 2004 aux États-Unis ${ }^{1}$}

Les élections présidentielles américaines sont caractérisées à la fois par la longueur du processus électoral, qui s'étend sur un peu plus de deux ans, et par la complexité que leur confère leur caractère indirect. La conjonction de ces deux spécificités est en partie responsable d'une certaine désaffection de l'opinion qui donne lieu à l'élaboration de stratégies capables de remobiliser un corps électoral perçu comme fragmenté, voire anomique. Or, depuis le début de la diffusion de masse d'Internet au milieu des années quatre-vingt-dix, le réseau a fait figure, dans les discours qui ont accompagné sa diffusion, de vecteur potentiel d'accroissement de la participation citoyenne prise au sens large, et de la participation électorale en particulier. Àce titre, il a rapidement été intégré dans le répertoire d'action des partis politiques. Ces derniers ont mis en place des sites très proches, par leur structuration interne et leur fonctionnement, de la documentation partisane la plus traditionnelle, servant de support à de fructueuses collectes de fonds. Ainsi, lors des législatives de mi-mandat qui se sont déroulées en 1998, plus des deux-tiers des candidats au Sénat et à la Chambre des représentants avaient mis en ligne un site de campagne, dont un tiers comprenait un dispositif de recueil de dons ${ }^{2}$. De la même façon, le comité de campagne de George W. Bush a levé plus de six millions de dollars de contributions de militants lors de la présidentielle de l'année $2000^{3}$. En dépit de ces succès, ni les républicains ni les démocrates n'ont mis en œuvre, sur leurs sites de campagne, le forum qui aurait pu fournir la dimension participative pourtant ostensiblement tant souhaitée ${ }^{4}$.

1. Une version préliminaire de cet article a fait l'objet d'une communication lors du $44^{\mathrm{e}}$ Congrès de la Société des anglicistes de l'enseignement supérieur (SAES), Université de Versailles/SaintQuentin-en-Yvelines, 27-29 mai 2004.

2. D. A. Dulio, D. L. Goff, J. Thurber, 1999, «Untangled Web: Internet use during the 1998 election », PS : Political Science and Politics, $\mathrm{n}^{\circ} 32$ (1), apsanet.org, p. 53-58.

3. W. Wilson, 2002, "A history of the 2000 presidential ecampaign of George W. Bush », V. Serfaty éd., L'Internet en politique, des États-Unis à l'Europe, Strasbourg, Presses universitaires de Strasbourg, p. 43-74.

4. E. C. Kamarck, 2002, "Political campaigning on the Internet: business as usual ? », E. C. Kamarck, J.S. Nye Jr. éd., Governance.com : Democracy in the Information Age, Washington DC, Brookings Institution Press, p. 81-103.

Université de Marne-la-Vallée, viviane.serfaty@univ-mlv.fr 
À ce constat de continuité, l'élection présidentielle de 2004 a cependant apporté un démenti, puisque la technologie du blog y a fait une entrée remarquée, à laquelle maintes propriétés novatrices ont été attribuées. Si ces affirmations sur leur caractère prétendument révolutionnaire, empreintes de déterminisme technologique, paraissent aussi répétitives qu'infondées, il est néanmoins légitime de se demander si, lors de la course à la présidence en 2004, aux ÉtatsUnis, l'usage des blogs a modifié les pratiques des acteurs de la sphère politique, qu'ils soient candidats, militants ou commentateurs. Les blogs possèdentils une dimension qui serait à même d'influencer le politique? La réponse à cette interrogation passera, dans un premier temps, par l'analyse du fonctionnement des blogs, puis, dans un second temps, par celle de leur insertion dans la stratégie de campagne de Howard Dean, ce gouverneur du Vermont dont la tentative, en 2004, d'obtenir l'investiture du Parti démocrate en vue de l'élection présidentielle s'est soldée par un échec au profit de John Kerry. Le troisième volet de ce travail, enfin, en s'appuyant sur l'étude d'un cas précis, esquissera une synthèse de la place qu'ont occupée les blogs dans l'espace public nord-américain lors de la campagne pour la présidentielle de 2004.

\section{Fonctionnement}

Le mot «blog » est de création récente 5 . Faisant écho à la pratique du journal de bord traditionnellement tenu par les capitaines de navires (ship's log), la forme a d'abord été désignée par le mot « weblog », rapidement transformé en «blog $»^{6}$. Cette abréviation a elle-même été particulièrement féconde, produisant une forme verbale régulière ( $t o \mathrm{blog}$ ) et de nombreuses formes nominales, dont le néologisme blogosphère qui fait référence non seulement à l'ensemble des textes publiés sous ce format, mais encore aux liens dynamiques créés entre différents blogs par la pratique du référencement réciproque à l'aide de liens hypertextes. Cette constante des blogs est désignée par un autre néologisme, blogroll.

Le blog se caractérise par une grande simplicité d'accès, qui autorise tout un chacun à publier sur Internet du texte, des images ou du son sans disposer du moindre savoir technique, celui-ci étant entièrement pris en charge par les sociétés qui fournissent les logiciels nécessaires et en assurent la gestion, de même que la publication sur le réseau. À l'origine simples listes de liens annotés vers d'autres sites, les blogs permettaient, avant la mise en place de moteurs de recherche efficaces, de s'orienter dans le fouillis des contenus mis

5. Pour un historique circonstancié de cette forme, voir V. Serfaty, 2004, The Mirror and the Veil : An Overview of American Online Diaries and Blogs, Amsterdam, New York, Rodopi, p. 19-22.

6. R. Blood, 2000, "Weblogs: a history and a perspective», rebeccablood.net (consultation en février 2002). Voir également D. Winer, 2002, «The History of Weblogs », weblogs.com (consultation en juin 2002). 
en ligne. Cette fonction continue d'ailleurs d'exister. Ainsi, le site Technorati (technorati.com) fournit la liste de toutes les entrées de blogs par thèmes ainsi que le nombre de liens hypertextes attachés par d'autres blogs à une entrée donnée. De cette façon, il est possible à la fois d'effectuer une recherche thématique et de mesurer de façon empirique le degré de diffusion d'un thème donné.

Les commentaires personnels sur l'actualité ou encore l'écriture de soi ont rapidement et profondément modifié l'allure comme le sens de ce mode d'écriture. Aussi, de leurs commencements, les blogs ont surtout conservé l'usage du lien hypertexte, omniprésent. Celui-ci introduit un élément de discontinuité et d'hétérogénéité dans le discours, puisqu'il est de règle d'inclure non seulement des références à toutes les informations que l'on mentionne, mais encore les thèses que l'auteur du blog conteste ou combat. Une relation discursive antagonique s'installe ainsi au cœur du dispositif.

Outre la simplicité d'utilisation et les liens hypertextes, le blog possède un autre signe distinctif, dans la mesure où l'auteur concède généralement à ses lecteurs un espace d'écriture qui leur permet de réagir à toute entrée et de publier eux-mêmes leurs commentaires. Le nombre de ces derniers s'affiche automatiquement, de même que le nombre de liens hypertextes insérés dans d'autres blogs afin de signaler l'intérêt d'une entrée donnée. Cette dernière fonction est celle de trackback qui, en conjonction avec le nombre de commentaires, permet d'évaluer rapidement le degré de résonance d'un texte publié dans la blogosphère. Enfin, le blog est mis en ligne selon un ordre chronologique inversé, où l'entrée la plus récente apparait en premier, tandis que les textes plus anciens sont graduellement archivés. Les entrées quotidiennes comme les commentaires de lecteurs restent cependant accessibles à l'aide d'un lien hypertexte permanent.

Le blog permet ainsi de mêler des caractéristiques apparemment contradictoires: la permanence de l'archive et de l'écrit coexiste avec la rapidité de réaction à une information donnée, plutôt caractéristique de l'oral; l'unité de ton introduite par un auteur ou une institution se voit rompue par l'hétérogénéité créée à la fois par les liens hypertextes et les discours des lecteurs, enchâssés dans le texte d'origine; et dans le domaine politique, l'enjeu public d'une élection se trouve pris en charge par une parole intensément personnelle qui mobilise nombre d'éléments informels, voire ludiques. Les blogs reproduisent donc un type d'énonciation qui tend à se constituer en norme sur Internet, et dont les caractéristiques saillantes sont l'oralisation de l'écrit, l'hétérogénéité et l'approche ludique. À celles-ci, ils adjoignent une forte dimension interactive (Serfaty, 2004, p. 66-69).

En effet, sous leur forme traditionnelle, les manifestes ou les discours sont avant tout à sens unique: qu'ils soient prononcés à la radio ou à la télévision, publiés dans les journaux ou sur un site Internet classique, il s'agit de communications émises par la hiérarchie du parti en direction des militants et du public; 
les réactions que ces discours suscitent restent essentiellement du domaine privé. Le logiciel du blog, par contre, autorise la mise en place d'un espace dialogique qui relie l'acteur politique à ses partisans de façon claire, mais permet aussi aux militants d'engager la conversation entre eux. Le risque n'est pas anodin, car le parti politique renonce alors volontairement à contrôler une partie de son territoire virtuel, d'ailleurs quelquefois envahi par les spam ou les commentaires, à l'occasion injurieux, de ses adversaires. Mais lorsque les militants s'impliquent par les textes brefs qu'ils mettent en ligne, ils créent un discours parallèle au ton informel, dépourvu de toute sanction officielle et donc autonome ${ }^{7}$. Ce degré d'autonomie est précisément ce qui à la fois pose problème aux hiérarchies partisanes et attire une frange neuve de militants.

\section{Militants, partis politiques et blogs}

Les blogs politiques ont commencé à proliférer aux États-Unis à la suite du succès rencontré en aout 2003 par la campagne de Howard Dean, candidat malheureux à l'investiture du Parti démocrate lors des primaires de 2004, mais figure de proue des campagnes électorales sur le Net.

Rappelons brièvement que H. Dean, le gouverneur démocrate du Vermont, unÉtat de petite taille plutôt orientéà gauche, est un homme politique peu connu lorsqu'il annonce, en décembre 2002, son intention de tenter l'aventure de l'investiture $^{8}$. S'il commence à être considéré comme un candidat sérieux dans le courant de l'été 2003 , c'est certes en raison de ses prises de position contre la guerre en Irak, mais aussi parce qu'il a su utiliser Internet de trois façons principales. Tout d'abord, par l'intermédiaire de son site officiel ${ }^{9}, \mathrm{H}$. Dean a levé plus de 51 millions de dollars constitués de petites sommes envoyées par des donateurs individuels, et non par des associations ou des groupes de pression ${ }^{10}$. Il a ainsi démontré qu'Internet pouvait mobiliser la base et, partant, se substituer aux modes de financement traditionnels des partis. Ces fonds ont cependant été utilisés principalement pour financer des campagnes à la télévision et des opérations de publipostage (Purdum, 2004), illustrant par là le fait qu'Internet s'agrège aux outils communicationnels plus anciens sans les supplanter.

D’autre part, H. Dean s'est appuyé sur un site Internet indépendant, MeetUp (meetup.com), pour organiser des rencontres entre partisans de sa campagne

7. Ainsi, l'entrée du 26 novembre 2003 a généré 174 commentaires, dont un grand nombre poursuit une conversation à plusieurs entre militants (blogforamerica.com, consultation en mai 2004).

8. T.S. Purdum, 2004, "The Dean comet: so what was that all about?", The New York Times, 22 février.

9. Voir sur deanforamerica.com (consultation en décembre 2004). Les archives de ce site sont toujours accessibles en janvier 2006.

10. Source: Federal Election Commission, chiffres au 31 mars 2004, après l'annonce du retrait de H. Dean le 18 février 2004 (herndon2.sdrdc.com, consultation en décembre 2004). 
dans tout le pays. Dans ce cas également, H. Dean a su détourner un site de son usage premier; en effet, établi au départ pour mettre en relation des personnes ayant des intérêts ou des gouts communs, MeetUp a vocation à s'intégrer avant tout à la culture populaire en y créant des réseaux sociaux. Son utilisation à des fins politiques allie adroitement culture politique plutôt élitiste et techno-culture populaire. Le répertoire des techniques de mobilisation de l'électorat, ainsi enrichi, a permis d'atteindre un segment plus large d'électeurs potentiels, parmi lesquels se sont trouvés, selon Reeher et Davis, nombre de primo-accédants à l'action politique ${ }^{11}$.

Enfin, l'usage du blog dans la campagne de H. Dean comportait deux volets: d'une part, un blog officiel, sur le site du candidat lui-même, et d'autre part des blogs de militants individuels. Sous l'influence de Joe Trippi, le directeur de campagne de H. Dean, le blog officiel était alimenté en partie par le comité directeur, qui y contribuait directement de façon occasionnelle, au lieu de laisser la plume uniquement à des bénévoles ou à des professionnels de la communication. Cette forme de participation directe des cadres politiques, qui apparaissait à intervalles irréguliers sur Blog for America (blogforamerica.com), ainsi que sur le site parallèle Dean Nation (dean2004.blogspot.com), a suscité l'enthousiasme de jeunes volontaires qui ont été particulièrement nombreux non seulement à intervenir dans les débats, mais encore à créer leurs propres blogs de soutien à sa campagne ${ }^{12}$.

La forte mobilisation de la base constitue la principale originalité des pratiques créées par les partisans de H. Dean. En effet, si tous les candidats en lice possédaient un site muni d'un blog, les entrées y étaient rédigées par un chargé de communication, et le texte mis en ligne ne constituait guère plus qu'une reproduction de la documentation de campagne de chaque parti. L'absence de voix narrative clairement reconnaissable retirait à ces blogs tout le bénéfice que leur usage du discours informel aurait pu leur apporter et les identifiait au produit d'une stratégie de communication bien plus qu'à une parole individuelle.

Les partisans de H. Dean, par contre, se sont personnellement impliqués dans ce mode de communication et les blogs, mis en place par des militants autonomes, n'ont plus qu'un lointain rapport avec les documents issus de la communication politique traditionnelle. En effet, la particularité de ces textes militants est avant tout de mêler le personnel et le politique, puisque les descriptions d'évènements privés y côtoient des mots d'ordre de mobilisation et des prises de position partisanes, qui quelquefois même semblent passer à l'arrière-plan ${ }^{13}$. L'ensemble de ces échanges, poursuivis sur une longue durée,

11. G. Reeher, S. Davis, 2004, "The political life of the Internet », The Responsive Community, $\mathrm{n}^{\circ} 14$ (Fall 2004), 2-3, amitai-notes.com (consultation en décembre 2004).

12. M. L. Sifry, 2004, "The rise of open-source politics ", The Nation, 22 novembre.

13. Voir par exemple annatopia.com, entrées du 2 juillet 2003 et du 26 juillet 2004 ; voir également blogforamerica.com, commentaire du 29 aout 2003 publié par Brian (consultation en mai 2004). 
tisse sur le Net des liens interpersonnels qui se voient renforcés par l'organisation sur le terrain, sans aucune intervention des directeurs de la campagne, de toutes les opérations de démarchage de porte-à-porte destinées à encourager les électeurs à soutenir H. Dean lors des primaires. L'action politique s'appuie ainsi sur une sociabilité militante qui, à la fois, découle des interactions sur Internet et finit par constituer l'une des conditions de cette action.

\section{Blogs de commentaire politique}

L'implication personnelle, par le biais des blogs, de la base militante du Parti démocrate et de nouveaux venus à la participation politique est cependant loin de rendre compte de l'ensemble des pratiques suscitées par ce mode d'écriture. En effet, le fort battage médiatique autour des partisans de H. Dean et de leurs blogs, en 2003, s'est accompagné d'une progression importante des blogs consacrés aux commentaires politiques, qui ont gagné une audience considérable à la faveur des élections. Selon une étude des sources d'information les plus utilisées par l'électorat américain au cours de la présidentielle de 2004, $30 \%$ des utilisateurs du réseau ont lu, pendant la campagne, des sites d'information alternatifs parmi lesquels figurent les blogs ${ }^{14}$. Sachant que $63 \%$ de la population adulte utilise Internet de façon régulière ${ }^{15}$, on peut estimer à environ trente-huit millions l'audience potentielle cumulée des blogs de commentaire politique. L'importance de ces chiffres semble indiquer que les blogs dépassent le champ étroit du militantisme pour s'insérer durablement dans les pratiques d'une proportion importante de la population.

Est-ce à dire que les blogs modifient suffisamment les pratiques pour permettre à ceux qui en étaient exclus d'accéder au champ politique? Un élément de réponse est fourni par le fait que de nombreux professionnels du journalisme se sont coulés avec bonheur dans ce nouveau mode d'écriture. Parmi les bloggers les plus lus, Andrew Sullivan (andrewsullivan.com), par exemple, a été rédacteur à l'hebdomadaire The New Republic et éditorialiste au New York Times Magazine et continue à collaborer à ces publications parmi bien d'autres. Mickey Kaus (kausfiles.com) a publié un ouvrage ainsi que des articles dans The New Republic et Newsweek; son blog se trouve maintenant hébergé sur le site du magazine Slate.com. Ainsi, loin d'être issues de la base, les célébrités de la blogosphère possédaient dès le départ des liens forts avec la sphère médiatique. Le blog a d'ailleurs été intégré aux pratiques journalistiques par de très

14. J. Horrigan, K. Garrett, P. Resnick, 2004, "The Internet and democratic debate », Pew Internet and American LifeProject, pewinternet.org, p. vii (consultation en décembre 2004).

15. M. Madden, L. Rainie, 2003, "America's online pursuits ", Pew Internet and American LifeProject, pewinternet.org, p. 3 (consultation en décembre 2004). Les auteurs estiment que la population adulte des États-Unis compte environ 200 millions de personnes. 
nombreux organes de presse écrite qui possèdent une version en ligne de leur publication.

Par ailleurs, nombre de blogs de renom sont dus à des universitaires en exercice qui, eux aussi, possèdent une maitrise certaine des codes de l'écriture. Glenn H. Reynolds (InstaPundit et glennreynolds.com) de même qu'Eugene Volokh (The Volokh Conspiracy) sont professeurs de droit, le premier à l'université du Tennessee, le second à l'université de Californie à Los Angeles (UCLA). D'autres universitaires s'essaient à ce mode conversationnel, comme Noam Chomsky (blog.zmag.org) ou bien Amitai Etzioni (amitai-notes.com) ${ }^{16}$, confirmant ainsi la montée en puissance de la légitimité des blogs.

Au vu de ces éléments, il n'est donc pas permis de conclure que l'usage des blogs modifie en profondeur les pratiques politiques. Les usages militants des blogs en font des outils de mobilisation efficaces doublés d'un outil de socialisation et de sociabilite militante qui, s'ils introduisent une variation, ne se démarquent pas fondamentalement de modes de communication antérieurs. En outre, les blogs individuels ne peuvent être considérés comme favorisant durablement l'accès au champ politique de catégories de la population qui en étaient auparavant exclues. Rédigés par des auteurs qui maitrisent pleinement les codes de l'écriture autant que ceux du débat politique, les blogs semblent occuper, dans les pratiques politiques comme dans l'espace public, une position autre, qu'il importe à présent de définir.

\section{Les blogs politiques dans l'espace public}

Le contenu politique des blogs étudiés, de même que leur fréquence de publication, semblent indiquer que c'est par rapport aux médias, et notamment à la presse écrite, que se définissent les rédacteurs de blogs. Pourtant, aucun des auteurs évoqués dans cette étude ne cherche à supplanter les médias traditionnels; tous reconnaissent le caractère limité de leur activité de collecte et de vérification de l'information ${ }^{17}$; les universitaires auteurs de blogs politiques notamment sont pleinement conscients du fait que leurs textes se situent hors de leur domaine de spécialisation. En dépit de ces affirmations, les blogs politiques les plus connus sont considérés comme des sources d'information et suscitent un nombre de visites quotidiennes qui, pour les dix premiers d'entre eux, s'élève de 30000 à $170000^{18}$, des chiffres qui, tout en restant très largement inférieurs

16. D. W. Drezner, H. Farrell, 2004, «The power and politics of blogs », communication au Congrès annuel de l'American Political Science Association, Chicago, 2-5 septembre, p. 14.

17. G. H. Reynolds, 2003, "Weblogs and journalism : back to the future ? » Nieman Reports, $n^{\circ} 57$ (3), p. 81-82, nieman.harvard.edu; P.Andrews, 2003, "Is blogging journalism?» Nieman Reports, $n^{\circ} 57$ (3), p. 63-64, nieman.harvard.edu (consultation en mai 2004).

18. Source : Site Meter, chiffres du 30 novembre 2004, cités par The Truth Laid Bear [sic], truthlaidbear.com (consultation en décembre 2004). 
à ceux de l'audience de la télévision, par exemple, n’en sont pas moins importants.

À cette situation, deux causes peuvent être trouvées. L'une d'entre elles tient aux modifications apportées par Internet à la sphère médiatique contemporaine, où les journalistes ne disposent plus du monopole de la fourniture de l'information et voient donc leur rôle de médiateurs contesté ${ }^{19}$. Les journalistes professionnels tirent leur légitimité de la déontologie qu'ils se sont donnée et qui repose sur les deux piliers de la véracité de l'information et de l'impartialité du journaliste ${ }^{20}$. Or, les blogs de commentaire politique semblent s'être donné pour mission tacite la surveillance sourcilleuse de la grande presse écrite ou télévisée, dans une posture qui tient à la fois de la contre-expertise et de la veille informationnelle et qui aboutit à l'ébranlement de l'autorité des médias. En dépit des dénégations des uns et des autres, un rapport de concurrence s'instaure entre médias traditionnels et blogs et aiguillonne l'intérêt pour ces derniers.

Ainsi, lors de la campagne électorale, Dan Rather, le grand reporter de la chaine de télévision CBS, présente des documents selon lesquels G. W. Bush aurait désobéi aux ordres lors de son service militaire mais que, l'influence de son père aidant, toute trace de son infraction aurait été retirée de son dossier militaire. L'authenticité des documents est immédiatement mise en cause sur le forum d'un site conservateur militant par un membre de l'armée de l'air, qui juge anachronique la mise en page des documents présentés à l'antenne et affirme: "Il faut que nous voyions ces rapports à nouveau! Ils ne sont pas mis en page selon le style que l'on utilisait lorsque je suis arrivé à l'armée de l'air. Ils ont plutôt le style et le format des documents que nous avons commencé à utiliser il y a environ douze ans (1992). Nos signatures étaient justifiées à gauche, maintenant elles sont plutôt à droite... comme celles qui viennent d'être montrées. » (Free Republic, freerepublic.com, entrée du 8 septembre 2004, ma traduction)

Les liens hypertextes reliant les blogs les uns aux autres assurent très rapidement une large audience à cette allégation, qui migre dès le lendemain de la blogosphère aux organes de presse traditionnels. Moins de deux semaines plus tard, le 20 septembre 2004, Dan Rather déclare publiquement qu'il ne peut garantir l'authenticité des documents et démissionne.

Ce que les blogs républicains appellent complaisamment «Rathergate » a certes été amplifié par le contexte préélectoral, mais indique néanmoins que les blogs politiques semblent avoir atteint une masse critique grâce à laquelle ils disposent d'un certain pouvoir de mise en agenda. En cela, ils concurrencent

19. N. Pélissier, 2002, «La plume dans la toile : l'identité des journalistes à l'épreuve des réseaux numériques », Médiamorphoses, $\mathrm{n}^{\circ}$ 4, p. 45-51.

20. M.-F. Bernier, 1994, Éthique et déontologie du journalisme, Sainte-Foy, Presses de l'Université de Laval, p. 119. 
directement la presse écrite et audiovisuelle, dont l'activité de sélection, de cadrage et d'interprétation de l'actualité est primordiale dans l'établissement de l'agenda politique. Les blogs les plus connus sont ainsi à même d'attirer l'attention sur des facettes de l'actualité peu ou mal représentées par les grands médias, contraignant ces derniers à tenir compte de la blogosphère - une évolution déjà largement entamée aux États-Unis.

La seconde raison qui sous-tend l'accroissement de l'audience des blogs de commentaire politique tient à la spécificité de la pratique discursive qui y est mise en place. Les médias traditionnels et, notamment, la télévision, tendent vers l'euphémisation du conflit en raison, entre autres, de l'exigence de rentabilité vis-à-vis des annonceurs. Les blogs politiques, par contre, privilégient l'engagement personnel. Par la radicalité délibérée de leurs prises de position, ils réintègrent dans la sphère publique une dimension de conflictualité très proche de celle du pamphlet. Les exemples d'Eschaton (atrios.blogspot.com), partisan des démocrates, ou du républicain John Hinderaker (powerlineblog. com), démontrent une polarisation très nette des positions respectives. Or, des vues aussi tranchées ne trouvent guère de place dans les médias, sinon sous forme de tribunes libres, généralement attribuées à des personnalités connues; si ces textes d'opinion provoquent des réactions de la part des lecteurs ou de l'audience, celles-ci sont soigneusement sélectionnées et formatées. Les blogs politiques, par contre, se construisent précisément sur leur vision partisane de l'actualité et sur les polémiques qu'ils instaurent et entretiennent dans une incessante confrontation idéologique. Aussi fuient-ils le consensus, même si l'on peut avancer, avec Daniel Drezner et Henry Farrell, qu'ils sont à même de générer un accord généralisé sur l'intérêt d'un évènement auquel acquiescent les médias traditionnels (Drezner, Farrell, 2004, p. 18).

Si le rôle des blogs est resté marginal lors de la campagne pour l'élection présidentielle américaine de 2004, ce mode d'écriture n'en a pas moins gagné en notoriété et en audience à la faveur de son adoption par l'un des candidats, mais aussi par la majorité des médias traditionnels. Le mode conversationnel élaboré sur les blogs de commentaire politique constitue un élément majeur de l'attirance qu'ils exercent. Les réactions rapides des auteurs de blogs à l'actualité, les commentaires en temps quasi-réel des lecteurs ainsi que leur ton informel se combinent pour constituer une norme discursive distinctive propre à cette écriture. Le texte qui en résulte est un texte collectif, car les lecteurs qui commentent ou qui signalent un élément de l'actualité, ou encore qui critiquent la façon dont les médias en rendent compte, participent à la production de l'information et à son interprétation. On ne saurait pourtant considérer que les blogs politiques concurrencent directement les journalistes. II semblerait 
plutôt qu'ils fonctionnent comme un niveau de médiation supplémentaire, qui élabore un schéma d'interprétation de l'actualité partisan et polémique et participe de façon ponctuelle à la mise en agenda de certains thèmes. C'est pourquoi les blogs politiques restent avant tout des outils de mobilisation dont la campagne 2004 a démontré l'efficacité et qui, en s'intégrant au répertoire d'action des militants comme de l'appareil des partis, ont entamé une diversification des pratiques politiques aux États-Unis.

\section{Références}

ALVAREZ M. R., HALLTh. E., 2004, Point, Click, Vote: The Future of Internet Voting, Washington DC, The Brookings Institution Press, $204 \mathrm{p}$.

ANDERSON D. M., CORNFIELD M. éd., 2003, The Civic Web: Online Politics and Democratic Values, Lanham, Rowman and Littlefield, $192 \mathrm{p}$.

BIMBER B., DAVIS R., 2003, Campaigning Online: the Internet in US Elections, Oxford, Oxford University Press, $224 \mathrm{p}$.

CORNFIELD M., 2004, Politics Moves Online: Campaigning and the Internet, New York, The Century Foundation Press, $120 \mathrm{p}$.

DAVIS R, 1999, The Web of Politics: The Internet s Impact on the American Political System, Oxford, Oxford University Press, $224 \mathrm{p}$.

_- à paraitre, Politics Online: Blogs, Chatrooms and Discussion Groups in American Democracy, Londres, Routledge, 2005, 176 p.

FOUNTAIN J., 2001, Building the Virtual State: Information Technology and Institutional Change, Washington DC, The Brookings Institution Press, 256 p.

GIMMLERA., 2001, "Deliberative democracy, the public sphere and the Internet», Philosophy and Social Criticism, $\mathrm{n}^{\circ} 7$ (27), 4, p. 21-39.

HILL K. A., HUGHES J. E., 1998, Cyberpolitics: Citizen Activism in the Age of the Internet, Lanham, Rowman and Littlefield, $224 \mathrm{p}$.

KAMARCKE., NYE J., 1999, Democracy.com: Governance in a Networked World, New York, Hollis Publishing, $221 \mathrm{p}$.

Nieman Reports, "Journalist's trade: weblogs and journalism», 2003, n5 57 (3), p. 6198.

NORRIS P., 2001, Digital Divide? Civic Engagement, Information Poverty and the Internet Worldwide, Cambridge, Cambridge University Press, $320 \mathrm{p}$.

PÉLISSIER N., 2002, "La plume dans la toile: l'identité des journalistes à l'épreuve des réseaux numériques », Médiamorphoses, ${ }^{\circ}$ 4, p. 45-51.

REYNOLDS G. H., 2003, «Weblogs and journalism: back to the future? » Nieman Reports, $\mathrm{n}^{\circ} 57$ (3), p. 81-82.

SERFATYV. éd., 2002, L'Internet en politique, des États-Unis à l'Europe, Strasbourg, Presses universitaires de Strasbourg, $423 \mathrm{p}$.

2002, "Forms and functions of conflict in online communities», Cercles, $n^{\circ} 5$, p. 183-197, http://www.cercles.com/n5/serfaty.pdf. 
2003, «La persuasion à l'heure d'Internet: quelques aspects de la cyberpropagande ", Vingtième siècle, $\mathrm{n}^{\circ} 80$, octobre-décembre, p. 123-131.

2004, "Online diaries: towards a structural approach», Journal of American Studies, $\mathrm{n}^{\circ} 38$ (3), p. 457-471.

2004, The Mirror and the Veil: An Overview of US Online Diaries and Blogs, Amsterdam, New-York, Rodopi, $154 \mathrm{p}$.

2005, article «Internet», Dictionnaire des élections européennes, Y. Déloye éd., Paris, Economica, 2005, p. 396-399.

SHANE P. M. éd., 2004, Democracy Online: The Prospects for Political Renewal Through the Internet, Londres, Routledge, $279 \mathrm{p}$.

WARSCHAUER M., 2003, Technology and Social Inclusion: Rethinking the Digital Divide, Cambridge, The MIT Press, $272 \mathrm{p}$.

WEBSTER F., 2001, Culture and Politics in the Information Age: A New Politics? Londres, Routledge, $231 \mathrm{p}$. 